\title{
Constitutive expression and roles of interleukin-8 in canine hemangiosarcoma
}

\author{
Jong-Hyuk Kim ${ }^{1,2}$, Katie L Anderson ${ }^{1}$, Aric M Frantz ${ }^{1,2}$, Ashley J Graef ${ }^{1,2}$, Milcah C Scott ${ }^{1,2}$, Leslie C Sharkey ${ }^{1,2}$, \\ Timothy D O'Brien ${ }^{2,3}$, Erin B Dickerson ${ }^{1,2}$, Jaime F Modiano ${ }^{1,2^{*}}$ \\ From São Paulo Advanced School of Comparative Oncology \\ Águas de São Pedro, Brazil. 30 September - 6 October 2012
}

\section{Background}

Interleukin-8 (IL-8) is a pleotropic cytokine that promotes tumor cell proliferation and survival, inflammation, and angiogenesis; however, its role in the pathogenesis of canine hemangiosarcoma (HSA) is unknown.

\section{Materials and methods}

Six canine hemangiosarcoma (HSA) cell lines and 24 primary and metastatic HSA tumor tissues were used to investigate the biological functions of IL-8. Roles of IL-8 were examined by analyzing microarray data, qRT-PCR, ELISA, MTS cell proliferation assay, and sphere-forming assay.

\section{Results}

IL-8 mRNA expression was variable among the tissue samples and both IL-8 mRNA and protein were variable among the cell lines. In contrast, IL-8 receptor mRNA and protein showed minimal variance. "IL-8 high" and "IL-8 low" groups were defined from the HSA tumor samples based on gene expression profiles. The "IL-8 high" group was associated with a "reactive microenvironment," showing enrichment of coagulation, inflammation, and fibrosis networks. However, IL-8 added exogenously and IL-8 blockade using neutralizing antibodies had no effect on HSA cell proliferation, despite apparent response to these signals at the level of gene expression. Similarly, neither addition nor blockade of IL-8 protected cells from apoptosis. IL-8 mRNA was elevated in HSA cancer stem cells, but exogenous IL-8 attenuated self-renewal of these cells.

\section{Conclusion}

The results of this study suggest that IL-8 is a driver of tumor heterogeneity, steering cells away from self-renewal and towards partial differentiation. It also could act to recruit (or produce from the tumor) inflammatory and pro-angiogenic cells to the microenvironment. We are testing this hypothesis in a robust xenograft model. These experiments will establish if IL-8 plays a role in progression and metastasis of canine HSA, and allow us to define the therapeutic potential of IL-8 blockade.

\section{Acknowledgements \\ This work was supported by grants CHF422, CHF 1131, and CHF 1429 from the AKC Canine Health Foundation.}

\section{Author details}

'Department of Veterinary Clinical Sciences, College of Veterinary Medicine, University of Minnesota, St. Paul, MN 55108, USA. ${ }^{2}$ Masonic Cancer Center, University of Minnesota, Minneapolis, MN 55455, USA. ${ }^{3}$ Department of Veterinary Population Medicine, College of Veterinary Medicine, University of Minnesota, St. Paul, MN 55108, USA.

Published: 4 April 2013

doi:10.1186/1753-6561-7-S2-P35

Cite this article as: Kim et al: Constitutive expression and roles of interleukin-8 in canine hemangiosarcoma. BMC Proceedings 2013 7(Suppl 2):P35.

\footnotetext{
* Correspondence: modiano@umn.edu

'Department of Veterinary Clinical Sciences, College of Veterinary Medicine, University of Minnesota, St. Paul, MN 55108, USA

Full list of author information is available at the end of the article
}

(c) 2013 Kim et al; licensee BioMed Central Ltd. This is an Open Access article distributed under the terms of the Creative Commons 